\title{
Oncolytic viruses: methods and protocols
}

\author{
DH Kirn, T-C Liu, SH Thorne (Editors) \\ Publisher: Humana Press, New York, Dordrecht, \\ Heidelberg, London. ISBN: 978-1-61779-339-4, \\ Hardcover: £85.50; eBook: £79.99
}

This volume that has been edited by pioneers in the field of oncolytic virus therapy represents an attempt to focus on specific methodologies and protocols that are currently being widely applied in this area of research. As such, it comprises a collection of 15 chapters that span a little less than 250 pages. The editors and the contributing authors are to be commended for covering so much ground in so little space and with such authority.

Most of the major species of viruses that are currently at the forefront of clinical translation (e.g. herpes simplex virus, adenovirus, reovirus, vaccinia virus and measles virus) are discussed, whereas others that are currently making their first tentative steps towards clinical studies (e.g. vesicular stomatitis virus (VSV) and myxoma virus) also receive attention. It is important to stress the fact that this volume will not provide a primer for novices in the field, as each chapter contains only the briefest of reviews of the biology and mechanism of tumour selectivity of each viral agent. Instead, this book should be regarded as a "How I do it" guide to practical science with oncolytic viruses. The 'Notes' sections at the end of each chapter are a particularly useful source of guidance that will assist researchers in establishing new methods or in trouble-shooting problematic techniques.

Many of the chapters focus on viral production and purification, but the text often usefully deals with technological approaches that endow the viruses that are made with specific, potentially beneficial properties. For example, there are two useful chapters on the production of capsid-modified or armed oncolytic adenoviruses by the groups led by Ramon Alemany and Dmitry Shayakhmetov, respectively. These chapters contain very useful step-by-step guides on the production of recombinant adenoviruses in yeast and bacteria. The authors provide a number of invaluable tips that are frequently absent from handbooks provided with commercially available kits. The guidance on more generic procedures, such as intravenous administration of viruses in mice and analysis of pharmacokinetics are less helpful and could reasonably have been omitted. The chapter on oncolytic VSV by John Bell will be of interest to many readers, especially given the fact that this agent is not permitted in some regulatory jurisdictions (e.g. United
Kingdom) because of its perceived threat to the farming industry. The text by Eva Galanis's group on oncolytic measles virus provides an authoritative state-of-the-art account of the manipulation of this agent in the laboratory.

A series of three chapters provide insights into methodologies for studying viral gene expression directly in tissue (Hallden) or by non-invasive means using luciferase (Weaver) or radioisotopic PET (Vassaux) imaging. Each of these chapters contains a great deal of practical information, but some of it is specific for the individual virus species or imaging platform used by the author. For example, the information on immunohistochemical analysis is only applicable to adenoviral vectors and the data on luciferase imaging is largely geared towards users of a single imaging system. Although these issues are difficult to avoid, the relatively narrow scope of the text on studying viral gene expression will leave a number of authors rather dissatisfied.

There are two chapters dealing with oncolytic adenoviruses and stem cells, each of which focuses on targeting the central nervous system. The first of the chapters addresses the concept of using neural stem cells to home to brain tumours and, thus, deliver a cargo of oncolytic adenovirus. This chapter contains repetition of aspects of adenovirus production that might have been removed with tighter editorial oversight. Nonetheless, the sections on preparing and maintaining neural stem cells and loading them with viral vectors will be of interest to many researchers. In chapter 9 , methodologies for targeting brain tumour stem cells with oncolytic adenoviruses are discussed in detail. There are helpful accounts of establishing neurosphere cultures and of profiling expression of tumour cell markers in vitro. Despite the merit of these individual chapters, one is left with the feeling that they do not necessarily fit within the volume as a whole.

Overall, this is not a volume that will be read cover-to-cover by many. Instead, it should be regarded as an eclectic collection of essays that will be extremely useful to a relatively small number of dedicated researchers in the field. Because of the relatively narrow focus on a limited number of viruses (the descriptions of techniques involving adenoviruses predominate), it is likely that many readers will be left disappointed. Even so, this volume should find a place in the library of most institutions involved in virological research.

Kevin J Harrington ${ }^{1}$

${ }^{1}$ The Institute of Cancer Research, Chester Beatty Laboratories, 237 Fulham Road, London SW3 6JB, UK 